\title{
Correction to: Zinc-Solubilizing Bacteria-Mediated Enzymatic and Physiological Regulations Confer Zinc Biofortification in Chickpea (Cicer arietinum L.)
}

\author{
Sadia Batool ${ }^{1} \cdot$ Hafz Naeem Asghar ${ }^{1}$ Muhammad Asif Shehzad ${ }^{2}$ D . Sanaullah Yasin ${ }^{3}$ Muhammad Sohaib $^{4}$. \\ Fahim Nawaz ${ }^{2,5} \cdot$ Gulzar Akhtar $^{6} \cdot$ Khuram Mubeen $^{2} \cdot$ Zahir Ahmad Zahir $^{1} \cdot$ Muhammad Uzair $^{7}$
}

Published online: 26 July 2021

(c) Sociedad Chilena de la Ciencia del Suelo 2021

\section{Correction to : Journal of Soil Science and Plant Nutrition https://doi.org/10.1007/s42729-021-00537-6}

The following error was present under the heading of 2.4.2 PCR Amplification for Bacterial-Specific 16S rRNA Gene in this article as originally published:

Wrong statement: Forward primer sequence was 5'-CAGCAGCCGCGGTAATAC-3' and reverse primer sequence 5'-CAGCAGCCGCGGTAATAC-3'.

Correct statement: Forward primer sequence was 5'ACGGGCGGTGTGTACAAG-3' and reverse primer sequence 5'-CAGCAGCCGCGGTAATAC-3'.
The original article has been corrected.

Publisher's Note Springer Nature remains neutral with regard to jurisdictional claims in published maps and institutional affiliations.

The online version of the original article can be found at https:// doi.org/10.1007/s42729-021-00537-6

Muhammad Asif Shehzad

asifbukhari01@gmail.com; asif.shehzad@mnsuam.edu.pk

Sanaullah Yasin

sanaullahyasin@gmail.com

Sadia Batool

sadiabatool1781@gmail.com

Hafz Naeem Asghar

naeemasghar@yahoo.com

Muhammad Sohaib

msohaib@ksu.edu.sa

Fahim Nawaz

fahim.nawaz@mnsuam.edu.pk

Gulzar Akhtar

gulzar.akhtar@mnsuam.edu.pk

Khuram Mubeen

khurram.mubeen@mnsuam.edu.pk

Zahir Ahmad Zahir

zazahir@yahoo.com
Muhammad Uzair

uzairshakoor@gmail.com

1 Institute of Soil and Environmental Sciences, University of Agriculture, Faisalabad 38040, Pakistan

2 Department of Agronomy, MNS University of Agriculture, Multan 66000, Pakistan

3 Central Cotton Committee, Cotton Research Station Ghotki, Multan 66000, Pakistan

4 Department of Soil Science, College of Food and Agricultural Sciences, King Saud University, Riyadh 11451, Saudi Arabia

5 Institute of Crop Science (340h), University of Hohenheim, 70599 Stuttgart, Germany

6 Department of Horticulture, MNS University of Agriculture, Multan 66000, Pakistan

7 School of Life Sciences and Biotechnology, Shanghai Jiao Tong University, Shanghai 201101, China 